\title{
A Retrospective Study of Screening of Common Transfusion Transmitted Infections in the Blood Bank of a Tertiary Care Centre
}

Kirana Pailoor ${ }^{*}$, Murali Keshava S, Prajwith Rai, Olivia D'Cunha and Lakshmi C

Father Muller Medical College Mangalore, Karnataka, India

*Corresponding author: Kirana Pailoor, Associate Professor, Department of Pathology, Father Muller Medical College, Fr.Muller Road, Kankanady, Mangalore, Karnataka 575002, India, Tel: 919448953716, 08242222569; E-mail: dockirana@yahoo.co.uk

Received date: Jul 27, 2014, Accepted date: Apr 03, 2015, Publication date: Apr 06, 2015

Copyright: ( 2015 Pailoor K, et al. This is an open-access article distributed under the terms of the Creative Commons Attribution License, which permits unrestricted use, distribution, and reproduction in any medium, provided the original author and source are credited.

\begin{abstract}
Introduction: Transfusion of blood and blood components, as a specialized modality of patient management saves millions of lives worldwide each year and reduce morbidity. It is well known that blood transfusion is associated with a large number of complications, some are only trivial and others are potentially life threatening, demanding for meticulous pre-transfusion testing and screening particularly for transfusion transmissible infections (TTI). The priority objective of blood transfusion screening (BTS) is thus to ensure safety, adequacy, accessibility and efficiency of blood supply at all levels. The objective of the present study was to assess the seroprevalence and trend of transfusion transmitted infections (TTI) among voluntary and replacement blood donors in the blood bank of Father Muller Medical College Hospital, Mangalore.
\end{abstract}

Methods: A retrospective review of donors record covering the period between January 2008 to December 2012 was analysed and all samples were screened for HIV, HBsAg, HCV, syphilis and malaria. The data was analysed using Statistical Package for the Social Sciences (SPSS) software.

Results: The overall prevalence of $\mathrm{HIV}, \mathrm{HbsAg}, \mathrm{HCV}$, syphilis and malaria were $0.06 \%, 0.30 \%, 0.06 \%, 0.12 \%$ and $0.01 \%$ respectively. With the implementation of strict donor criteria and use of sensitive screening tests, it may be possible to reduce the incidence of TTI in the Indian scenario.

Conclusion: All blood donations should be screened for TTI's, thus ensuring safe blood supply to the recipients. With the implementation of strict donor selection criteria, use of sensitive screening tests and establishment of strict guidelines for blood transfusion it may be possible to reduce the incidence of TTI in the Indian scenario.

Keywords: Seroprevalence; Transfusion transmitted infections; Screeing for infections

\section{Introduction}

Transfusion of blood and its components is lifesaving as well as it has life threatening hazards. With every unit of blood there is a $1 \%$ chance of transfusion associated problems including transfusion transmitted diseases [1,2]. Preventing the transmission of infectious diseases through blood transfusion in developing countries is difficult given that the resources required are not always available even when policies and strategies are in place. These strategies have been extremely effective but transmission of diseases still occurs, primarily because of the inability of the test to detect the disease in the preseroconversion or 'window' phase of their infection, high cost of screening, a lack of funds and trained personnel, immunologically variant viruses, non-seroconverting chronic or immuno silent carriers and inadvertent laboratory testing errors. Transfusion Transmitted Infections (TTI) is still a major concern to patients, physicians and policy makers who wish to see a risk free blood supply.

In our study, we aimed to estimate the seroprevalence of HIV, HBV, HCV, Syphilis and Malaria among the blood donors .It gives an estimation of disease burden in the community. We have included five years in this study so as to get a better idea on the increasing or decreasing trends of TTI.

\section{Materials and Methods}

The present study was carried out in the blood bank of Father Muller Medical College Hospital, Mangalore. The records of blood donors who reported to blood bank from January 2008 to December 2012 were analyzed. The permission from head of the institution and clearance from Institutional Ethics Committee was obtained.

The donor questionnaire forms with details of donor and the results of serological tests are maintained in the blood bank. The venous blood is collected from all eligible donors after the complete physical examination by blood bank medical officer. The venous blood so collected is tested for HBsAg, HIV, HCV, Syphilis and Malaria. Two kits were used based on WHO recommendation of two different testing strategies involving enzyme-linked immunosorbent assay (ELISA) and/or simple or rapid assays for surveillance. VDRL test was used for screening of Syphilis. The positive VDRL test was confirmed by Treponema Pallidum Haemagglutination (TPHA) test. The malarial parasite was examined on peripheral blood smear and confirmed by serology. The blood was allowed to clot for 50 minutes at room temperature and serum was separated after centrifugation. The serum sample was then subjected to serological test. 
Citation: Pailoor K, Keshava SM, Rai P, D'Cunha O, Lakshmi C (2015) A Retrospective Study of Screening of Common Transfusion Transmitted Infections in the Blood Bank of a Tertiary Care Centre. J Blood Disorders Transf 6: 267. doi:10.4172/2155-9864.1000267

Page 2 of 3

In house positive and negative controls were performed for each reagent lot. The screening of blood donors or donated blood for HBsAg, HIV, HCV, VDRL and malaria is mandatory. Archived results from our hospital blood bank was used for this study with approval from the hospital authorities.

The data was analysed using Statistical Package for the Social Sciences (SPSS) software. Statistical tools like mean, frequency, chi square test and Fishers exact test were used. The prevalence was calculated at a $95 \%$ confidence interval and p value of 0.05 was considered to be significant.

\section{Results}

A total of 29,195 blood donors were screened from January 2008 to December 2012. Out of these, 27,990 (95.9\%) were males and
$1,205(4.1 \%)$ were females and 23,305 donations were voluntary and 5,890 were replacement donation. The number of donations has increased from 4,589 in 2008 to 5,432 in 2009 to 6,152 in 2010 to 6,103 in 2011 and 6,919 in year 2012. The results of seropositive samples for HBsAg, HCV, HIV, VDRL and Malaria are shown in Table 1. Analysis of the prevalence among males and females revealed no statistically significant variation in the occurrence of the infections among males and females (Table 2). The overall prevalence rate for HIV, HBV and Syphilis at our centre were highest in 2010, 2011, and 2011 respectively (Figure 1) [3].

\begin{tabular}{|c|c|c|c|c|c|c|}
\hline & Year 2008 & Year 2009 & Year 2010 & Year 2011 & Year 2012 & Total \\
\hline Total units & 4589 & 5432 & 6152 & 6103 & 6919 & 29195 \\
\hline HIV & $\begin{array}{l}3 \\
0.07 \%\end{array}$ & $\begin{array}{l}4 \\
0.07 \%\end{array}$ & $\begin{array}{l}6 \\
0.10 \%\end{array}$ & $\begin{array}{l}2 \\
0.03 \%\end{array}$ & $\begin{array}{l}2 \\
0.03 \%\end{array}$ & $\begin{array}{l}17 \\
0.06 \%\end{array}$ \\
\hline HBsAg & $\begin{array}{l}14 \\
0.31 \%\end{array}$ & $\begin{array}{l}20 \\
0.37 \%\end{array}$ & $\begin{array}{l}13 \\
0.21 \%\end{array}$ & $\begin{array}{l}28 \\
0.46 \%\end{array}$ & $\begin{array}{l}12 \\
0.17 \%\end{array}$ & $\begin{array}{l}87 \\
0.30 \%\end{array}$ \\
\hline Anti HCV & $\begin{array}{l}4 \\
0.09 \%\end{array}$ & $\begin{array}{l}5 \\
0.09 \%\end{array}$ & $\begin{array}{l}0 \\
0.0 \%\end{array}$ & $\begin{array}{l}4 \\
0.07 \%\end{array}$ & $\begin{array}{l}5 \\
0.07 \%\end{array}$ & $\begin{array}{l}18 \\
0.06 \%\end{array}$ \\
\hline VDRL & $\begin{array}{l}6 \\
0.13 \%\end{array}$ & $\begin{array}{l}3 \\
0.06 \%\end{array}$ & $\begin{array}{l}8 \\
0.13 \%\end{array}$ & $\begin{array}{l}10 \\
0.16 \%\end{array}$ & $\begin{array}{l}8 \\
0.12 \%\end{array}$ & $\begin{array}{l}35 \\
0.12 \%\end{array}$ \\
\hline Malaria & $\begin{array}{l}0 \\
0.0 \%\end{array}$ & $\begin{array}{l}1 \\
0.02 \%\end{array}$ & $\begin{array}{l}1 \\
0.02 \%\end{array}$ & $\begin{array}{l}1 \\
0.02 \%\end{array}$ & $\begin{array}{l}0 \\
0.0 \%\end{array}$ & $\begin{array}{l}3 \\
0.01 \%\end{array}$ \\
\hline
\end{tabular}

Table 1: Showing results of seropositive donors blood samples for HBsAg, HCV, HIV, VDRL and Malaria.

\begin{tabular}{|l|l|l|l|}
\hline Years & Positive & Negative & Total \\
\hline 2008 & 27 & 4562 & 4589 \\
\hline & $0.59 \%$ & $99.41 \%$ & \\
\hline 2009 & 33 & 5399 & 5432 \\
\hline & $0.61 \%$ & $99.40 \%$ & \\
\hline 2010 & 28 & 6124 & 6152 \\
\hline & $0.46 \%$ & $99.54 \%$ & \\
\hline 2011 & 45 & 6058 & 6103 \\
\hline & $0.74 \%$ & $99.30 \%$ & \\
\hline 2012 & 27 & 6892 & 6919 \\
\hline & $0.39 \%$ & $99.60 \%$ & \\
\hline
\end{tabular}

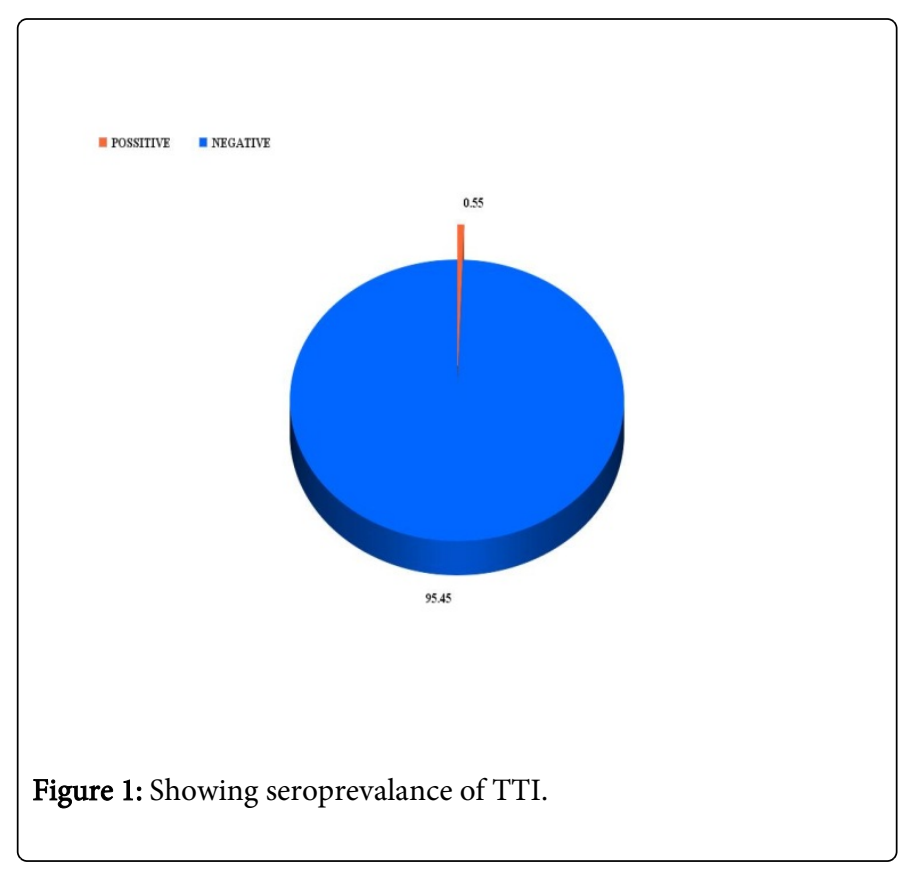

Table 2: Showing results for seropositive and seronegative blood samples from the year 2008 to 2012.

Figure 1: Showing seroprevalance of TTI. 


\section{Discussion}

Transfusion of blood and blood products is a life saving measure and helps innumerable people worldwide. At the same time however, blood transfusion is an important mode of transmission of infection to the recipients. The risk of TTI has declined dramatically in high income nations over the past two decades, primarily because of extraordinary success in preventing HIV and other established transfusion transmitted viruses from entering the blood supply. But the same may not hold good for the developing countries. The national policy for blood transfusion services in our country is of recent origin and the transfusion services are hospital based and fragmented .In developing countries the prevalence of TTI is much higher and quite far from attaining a zero risk level at the present moment. Majority of the donors were aged between 18 and 30 years which are in keeping with the age of college students who often volunteer called to donate at our institution. Other studies such as Fernandes et al. [4] have shown similar age distribution.

In this study we found that during the five year period, there was no significant increase or decrease in the prevalence of HIV, HBV, HCV, VDRL and malaria among the blood donors. This shows that the prevalence of these infections are still same as that of five years ago.

Seroprevalence of HIV is $0.06 \%$ in our study which is in contrast to other studies such as Arora et al. [5], Sawke et al. [6] and Mujeeb et al. [7] 1 which was observed to be $0.3 \%, 0.5 \%$, and $0 \%$ respectively. A WHO report states that the viral dose in HIV transmission through blood is so large that one HIV positive transfusion leads to death, on an average, after two years in children and after three to five years in adult. Hence, safe transfusion practices like avoidance of professional donors, promoting increased voluntary blood donation and practices of autologous blood transfusion should be encouraged.

Seroprevalence of HBsAg in this study was $0.30 \%$, which is very less compared with other studies such as Arora et al. [5], Sawke et al. [6] and Chandra et al. [8] which shows $1.7 \%, 2.9 \%$ and $5 \%$. Hepatitis B is a special problem in India since it is a medium endemic area. This problem is acutely reflected in blood transfusion services due to dependence on first-degree relative or paid blood donors and lack of non-remunerated repeat voluntary blood donors. Routine HBsAg screening in blood units does not eliminate the risk of $\mathrm{HBV}$ transmission. HBsAg test may be negative in the window phase of HBV infection, in the convalescence phase and also in HBV chronic infection, with very low level of viremia. Prevention of posttransfusion hepatitis starts with selection of non-remunerated voluntary blood donors [9].

Seroprevalence of HCV is 0.06 , which is less compared to other studies such as Kulkarni et al. [10]. Anti-HCV test has been made mandatory by the Government of India from June 2001.

This study highlights $0.12 \%$ prevalence of syphilis infection, which is high compared to study done by Kulkarni et al. [10] which showewd $0.04 \%$. But Pallavi et al. observed in their study a much higher prevalence of $0.28 \%$ with respect to syphilis [11]. In the recent years syphilis has also acquired a new potential for morbidity and mortality through association with increased risk of HIV infection, thus making safe blood more difficult to get. A strict selection criteria for blood donors to exclude those with multiple sexual partners is recommended and all the affected donors should be treated appropriately.
In addition we also found $0.01 \%$ prevalence of malaria. But most of the other studies such as Arora et al. [5], Sawke et al. [6] and Chandra et al. [8] showed no prevalence. More research from areas with diverse prevalence is needed to ascertain the true risk of acquiring malaria through transfusion of blood. Malaria occurring after a blood transfusion may have been acquired due to bite by a mosquito. So study designs have to incorporate molecular techniques which will help to differentiate between malaria acquired from mosquito bite and blood transfusion.

The low prevalence of transfusion transmitted infections rate in this study may be attributed to increased number of voluntary donors donating blood and aslo following strict screening criteria at our blood bank

Majority of the donors (98\%) were males, which is comparable to the study done by Kulkarni et al. [10], Rao, et al. [9] and Arora et al. [5]

\section{Conclusion}

All blood donations should be screened for Transfusion transmitted infections, thus ensuring safe blood supply to the recipients. With the implementation of strict donor selection criteria, use of sensitive screening tests such as neucleic acid testing and establishment of strict guidelines for blood transfusion it may be possible to reduce the incidence of Transfusion transmitted infections in the Indian scenario.

\section{References}

1. Fiebig EW, Busch MP (2008) Infectious disease screening. In: Technical manual. (16th edtn.). American Association of Blood Banks, 241-278.

2. Chandra T, Kumar A, Gupta A (2009) Prevalence of transfusion transmitted infections in blood donors: an Indian experience. Trop Doct 39: 152-154.

3. Srikrishna A, Sitalakshmi S, Damodar P (1999) How safe are our safe donors? Indian J Pathol Microbiol 42: 411-416.

4. Fernandes H, D'souza PF, D'souza PM (2010) Prevalence of transfusion transmitted infections in voluntary and replacement donors. Indian J Hematol Blood Transfus 26: 89-91.

5. Arora D, Arora B, Khetarpal A (2010) Seroprevalence of HIV, HBV, $\mathrm{HCV}$ and syphilis in blood donors in Southern Haryana. Indian J Pathol Microbiol 53: 308-309.

6. Sawke N, Sawke GK, Chawla (2010) Seroprevalence of common transfusion - Transmitted infections among blood donors. People's journal of scientific research 3: 5-7.

7. Mujeeb SA, Mehmood K (1996) Prevalence of HBV, HCV, and HIV infections among family blood donors. Ann Saudi Med 16: 702-703.

8. Chandra T, Kumar A, Gupta A (2009) Prevalence of transfusion transmitted infections in blood donors: an Indian experience. Trop Doct 39: 152-154.

9. Rao P, Annapurna K (1994) HIV status of blood donors and patients admitted in KEM Hospital Pune. Indian J Hemat Blood Transf 12: 174176.

10. Kulkarni N (2012) Analysis of the seroprevalence of HIV, HBsAg, HCV and syphilitic infections detected in the pretranfusion blood: A short report. International Journal of Blood Transfusion and Immunohematology 2: 1-3.

11. Pallavi P, Ganesh CK, Jayashree K, Manjunath GV (2011) Seroprevalence and Trends in Transfusion Transmitted Infections Among Blood Donors in a University Hospital Blood Bank: A five Year Study. Indian J Hematol Blood Transfus 27: 1-6. 